\title{
Proposal of a Digital Switch for Easy Changes of Number and Types
}

\author{
Ryota Iwaizono a, ${ }^{\text {, }}$, Mitsunori Mizumachi ${ }^{\text {a }}$, Seiichi Serikawa ${ }^{\text {a }}$ \\ ${ }^{a}$ Department of Electrical and Electronic Engineering, Kyushu Institute of Technology, Kitakyushu, Japan \\ *Corresponding Author: iwaizono.ryouta849@mail.kyutech.jp
}

\begin{abstract}
A digital type input device that allows the user to easily change the number of switches and the switch type has been proposed in this study. Even with the latest method, there was a limit to the number of switches that can be increased due to the influence of noise in actual environment, and the switch type was only touch. In addition, it was difficult for users to customize it freely because it was not possible to detect multiple switches at the same time. In this study, each switch is designed to be detected digitally in sequence. As the result, the limitation on the number of switches caused by the noise is released, and multiple switches can be detected at the same time. Furthermore, the switch type can be changed from touch panel to push button and sensor module. They can all be used with the same software because they all determine ON/OFF based on the pulse waveform in spite of different type switches. It was confirmed by simulation and experiment that the status of the switches can be detected correctly even if the switch type is changed. The proposed method also has an advantage over the conventional methods on the flexibility because the design, shape, size, surface condition, and switch type can be easily changed by using only two signal lines regardless of the number of switches.
\end{abstract}

Keywords: Input device, Sensing, I2C $\mathrm{C}^{(1)}$

\section{Introduction}

Recently, a variety of input device have been used for electrical appliances and devices to satisfy user demand. For examples, keyboards, mouse, push buttons, touch screens, joysticks, and so on, have been developed to make them more convenient. Most of such devices use push buttons and touch screens. Push-buttons have simple construction, so they are used for many control devices such as TV remote controls and keyboards. These are very useful, but the number and size of the buttons are fixed. The touch screen requires no pressure to press it. Therefore, it is utilized for devices used by an unspecified number of people, such as bank ATMs. In this way, the input type of the device that is most suitable for the intended use is selected. For conventional input devices, the manufacturer determines the input type. Input devices are generally sold by incorporating them into products. The number and type of switches of the input device cannot be changed after shipment. Input devices are not equally useful to everyone. For example, an elderly person who does not have power to push a button may be not easy to a push button. Depending on user, an input device built into a product may have poor operability. Therefore, some input devices have been developed that can be easily customized by user. A touch panel switch is proposed as one of them ${ }^{(2,3)}$. The touch panel switch allows the user to change the number of switches and the shape. This method measures the current flowed on touch panel switch. The analog value of current determines the state of on or off of the touch panel. The mechanism is simple and it has flexibility. The number of buttons can be changed easily, and the shape can be changed easily, so they can be attached not only to the flat plate but also to curved surfaces. For example, it is possible to use a wearable input device that is attached to the body, or many touch panels can be attached on a surface of a complex-shaped object. For conventional touch buttons, a signal line of the button is directly connected to the controller. Then, the number of signal lines increases according as the number of buttons increases. Practically, the number is 10 or 20 at most. On the other hand, the proposed touch panel can be easily expanded. The number of signal lines is 2 , even if the number of switches is increased infinitely in principle. However, the number of switches is limited. In practice, if the number of buttons increases up to 100 , the analog current flowing on the panel decreases and it cannot be detected by the noise. In addition, detection errors might occur when multiple panels are touched at the same time. Furthermore, 
since the input type is only touch, it is suitable for no power people but not suitable for healthy people not to get push sensitivity. Thus, touch panel switches are not always desirable as input device for everyone.

In this study, we propose a new digital type input device in order to improve the customizability of the input device. This method judges the state of on or off digitally. Therefore, the number of switches can be increased infinitely without affection of noise. In addition, even when some switches are turned on at the same time, they can be recognized correctly. Further, the type of switch can be easily changed by replacing the touch panel switch to push button and sensor module.

\section{Principle}

\subsection{Pulse Detection Type Touch Sensor}

A pulse detection type of touch sensor detects a capacitance obtained by current between human body and electrode. Figure 1 shows touch sensor circuit, and equivalent circuit of human body. The dotted line in Fig. 1 shows the resistance and capacitor, which is an equivalent circuit of the human body. Let the upper node of the resistor $\mathrm{R}$ be (a), and the lower node be (b) in Fig.1. A pulse is sent to the node (a), and the waveform of the node (b) is received. The ON/OFF of the touch panel is determined based on the delay time in case of rising of the waveform of the node (b). If there is no contact with the touch panel, the waveform of the node (b) will be the same as that of the node (a), and if there is contact, the waveform of the node (b) will rise delayed. The waveform at the time of the touching panel is shown in Fig.2, where the sent pulse of the node (a) and the received waveform of the node (b) are shown in the upper and lower panels, respectively. As understood from the dotted line in Fig. 1, the resistance and capacitor of the human body cause a delay waveform. In this study, the ON/OFF of touch is determined based on the delay time. Therefore, the threshold voltage Vth is set as shown in the lower waveform of Fig. 2. Let the time until the voltage of received the waveform reaches $V$ th be $T$. When the touch panel is not touched by a human, $\mathrm{T}=0$ because of no delay. On the other hand, when the touch panel is in contact, a time delay occurs, it is about $\mathrm{T}>10[\mathrm{~ms}]$. Thus, the pulse detection type of touch sensor can detect the contact by using delay time.

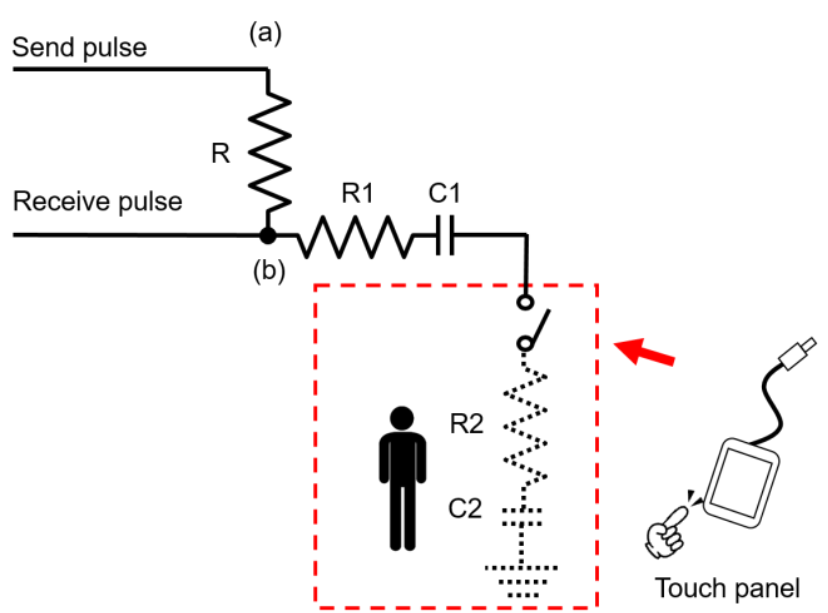

Fig. 1. Touch Sensor Circuit

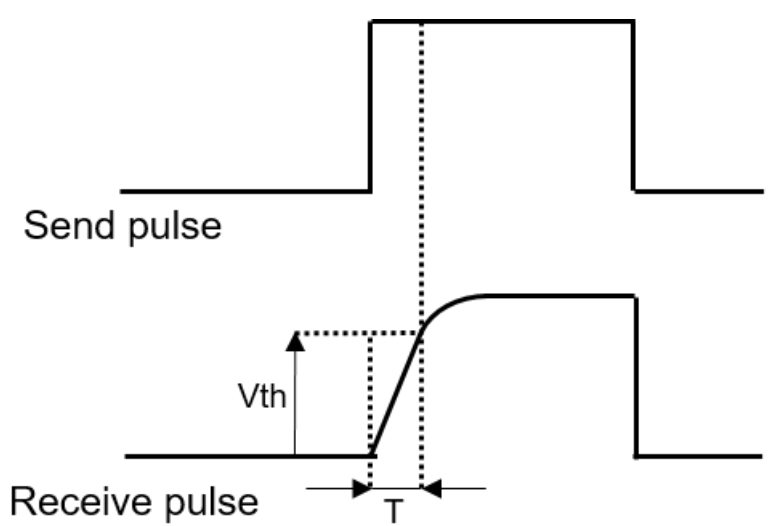

Fig. 2. Touch Sensor Signals

\subsection{Pulse Detection Type of Push Button}

Figure 3 shows the circuit diagram used for the pulse detection type of push button. This push button closes switch when pressed. As a result, resistor $\mathrm{R} 1$ and capacitance $\mathrm{C} 1$ are connected to form a delay circuit. Therefore, the same waveform as in Fig. 2 can be obtained with the push button. Let the upper node of the resistor R be (a), and the lower node be (b) in Fig.3. A pulse is sent to the node (a), and the waveform of the node (b) is received. The ON/OFF of the push button is determined based on the delay time in case of rising of the waveform of the node (b). If the push button is not pressed, the waveform of the node (b) will be the same as that of node (a), and if it is pressed, the waveform of the node (b) will rise delayed. As the same analogy of in Chapter 2.1 , the pulse detection type of push button can detect the state of the button by delay time. 


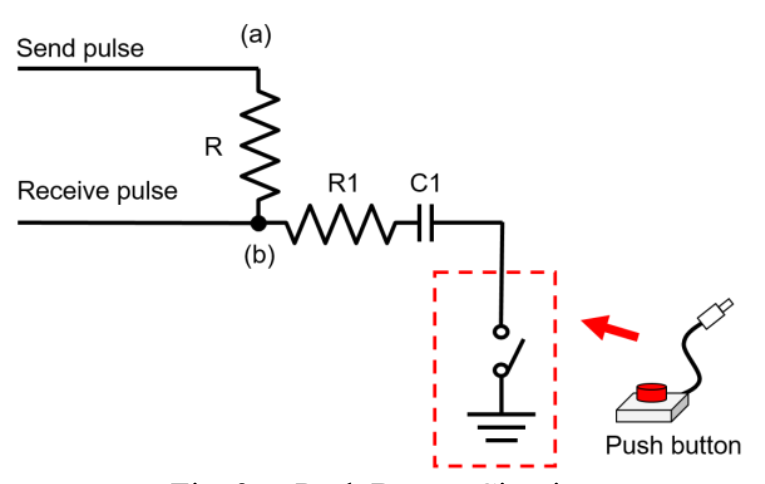

Fig. 3. Push Button Circuit

\subsection{Pulse Detection Type of Sensor module}

Figure 4 shows sensor module circuit. The sensor module used in this study outputs two states, one is High and another is Low. When the output of this sensor module is High, a high voltage is applied to gate $(\mathrm{G})$ of MOSFET. and current flows between the drain (D) and source (S). As a result, the resistor $\mathrm{R} 1$ and the capacitance $\mathrm{C} 1$ are connected to form a delay circuit. Therefore, the same waveform as in Fig. 2 can be obtained with the sensor module. Let the upper node of the resistor R be (a), and the lower node be (b) in Fig.4. A pulse is sent to the node (a), and the waveform of the node (b) is received. The ON/OFF of the sensor module is determined based on the delay time in case of rising of the waveform of the node (b). When the sensor module is Low, the waveform of the node (b) will be the same as that of node (a), and if it is High, the waveform of the node (b) will rise delayed. As analogy in Chapter 2.1, the pulse detection type of sensor module can detect the state of ON/OFF by delay time.

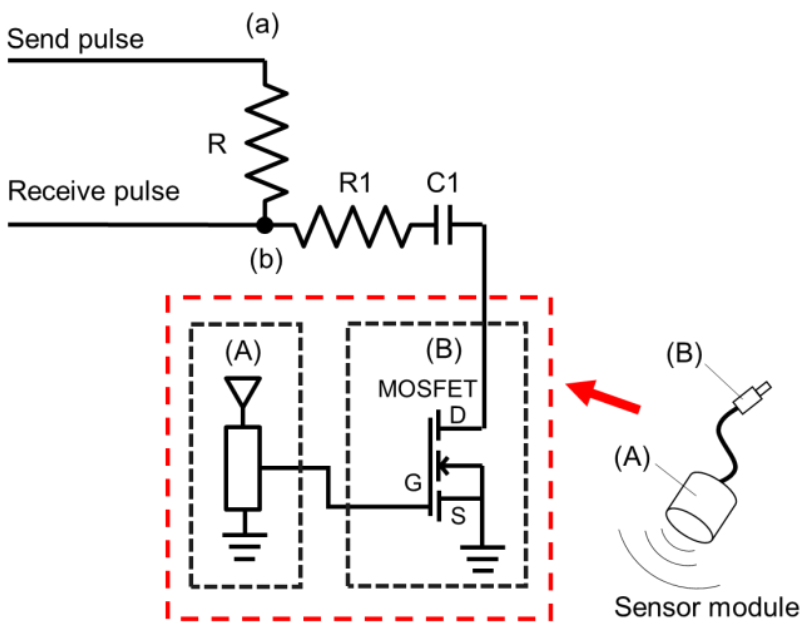

Fig. 4. Sensor Module Circuit

\section{Proposed Method}

\subsection{Summary of proposed method}

Figure 5 shows the diagram of the proposed input device in this study. The input device in this figure consists of a detection part and an input part. the detection part includes a CPU(A), signal lines(B), expanders(C), and the input part includes an element(D). The CPU(A) and expanders(C) are connected in parallel by two signal lines(B), so that expander $(\mathrm{C})$ can be easily added. The expander(C) is connected to many elements(D). The element(D) is a touch panel or push button, sensor module. One expander is connected to $\mathrm{N}$ elements. In our experiment, $\mathrm{N}=8$.

Next, the role of each part is explained as follows. The CPU(A) directly detects the conditions of ON/OFF of all elements(D) through signal lines(B) and expander (C). The $\mathrm{CPU}(\mathrm{A})$ communicates to expander(C) by $\mathrm{I} 2 \mathrm{C}$ communication on signal lines(B). A microcomputer (Arduino, Arduino UNO Rev3) is used as a CPU(A). It has the role of a master in $\mathrm{I} 2 \mathrm{C}$ communication. As an expander(C), an I/O expander (Microchip, MC23017) is used. This has the role of slave in $\mathrm{I} 2 \mathrm{C}$ communication. The $\mathrm{I} / \mathrm{O}$ ports of the expander are independent, so the noise has no effect to the other I/O ports when the number of I/O port increases. Therefore, an user can add a switch without considering noise. Since all of these elements (D) use the pulse detection type, any element can be operated with the same program.

\subsection{Detection Algorithm}

Figure 6 shows a detection algorithm. The flowchart (A)

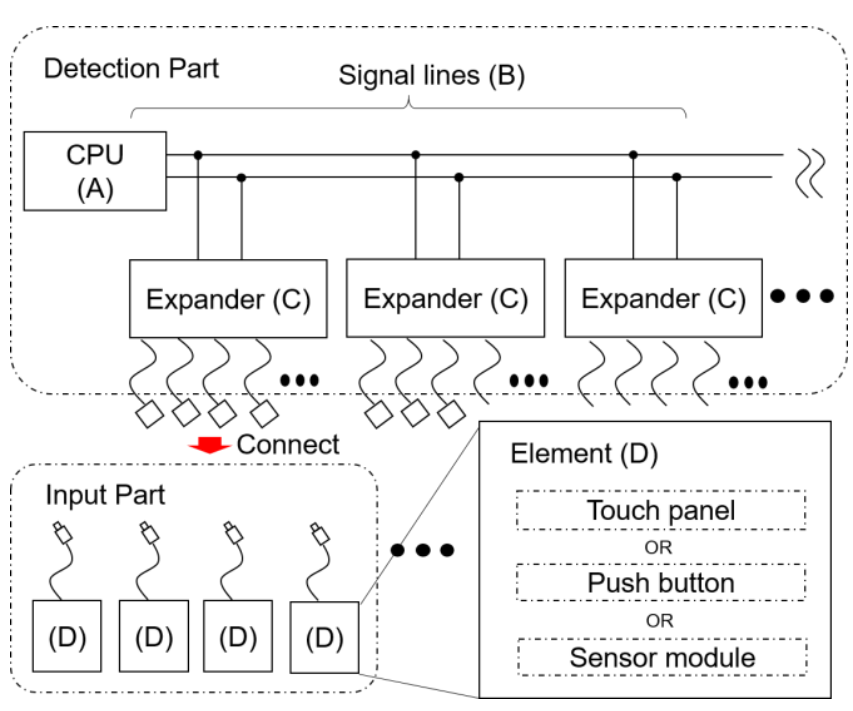

Fig. 5. Input Device Diagram 
shows the overall flow. The flowchart (B) shows an algorithm to detect ON/OFF of one port. The flowchart (A) means that the flowchart $(\mathrm{B})$ is repeated for all the connected expanders and the elements. In the flowchart (B), the delay time is measured, and the ON/OFF of the element is determined based on the delay time.

At first, the detail of the flowchart (A) is explained as follows.

(1) Loop (a) is paired with (5). Repeats (2) and the subsequent steps for all expanders.

(2) Loop (b) is paired with (4). Repeats (3) and the subsequent steps for all elements.

(3) Judgment process, call flowchart (B).

(4) End condition of loop (2).

(5) End condition of loop (1).

Next, the flowchart (B) will be described as follows. The flowchart means that it measures delay time and judges ON/OFF.

(1) Send a pulse to the node(a) in Fig.2.

(2) Loop (c) is paired with (4). Repeats (3) and subsequent steps until the end of the pulse.

(3) Receive the waveform of the node(b) in Fig.2.

(4) End condition of the loop (2).

(5) Measure whether there is a delay or not.

(6) Judge ON/OFF of the element.

Thus, the condition of ON/OFF to the element can be judged.
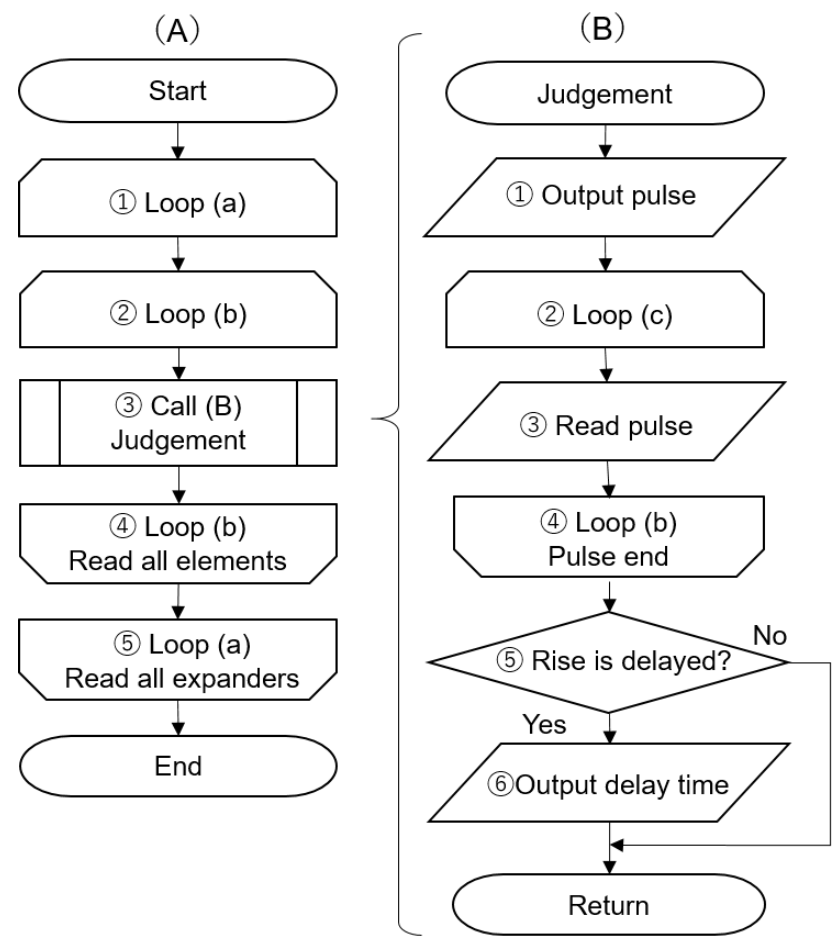

Fig. 6. Detection Algorithm

\section{Experiment}

\subsection{Validity of proposed method}

In this experiment, the validity of the proposed method is verified by both simulation and experiment. Waveform measurement is performed by replacing the element with a touch panel, push button, or sensor module.

\subsection{Simulation}

Figure 7 shows the circuit used for the touch panel simulation. The voltage of the pulse signal is set to be $5[\mathrm{~V}]$, the rising time is set to be $0.001[\mathrm{~ms}]$, the falling time is set to be $0.001[\mathrm{~ms}$ ], the pulse width is set to be $15[\mathrm{~ms}]$, and the pulse off width is set to be $15[\mathrm{~ms}]$. The delay circuit sets the resistance to $100[\Omega]$ and the capacitance to $4700[\mathrm{pF}]$. The resistance and capacitance of the human body are regarded to $400[\Omega]$ and $680[\mathrm{pF}]$ respectively. Figure 8 shows the obtained waveform. Figure 9 shows the circuit used for the push button simulation, and Figure 10 shows the obtained waveform. Figure 11 shows the circuit used for the sensor module simulation, and Figure 12 shows the obtained waveform. From the simulation results, we can confirm that there is a delay in the rise time. This makes it possible to

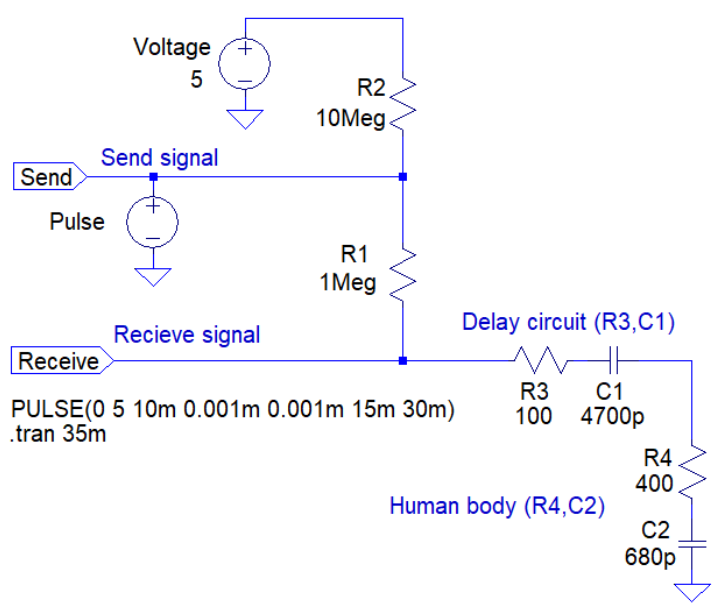

Fig. 7. Simulation Circuit (Touch Panel)

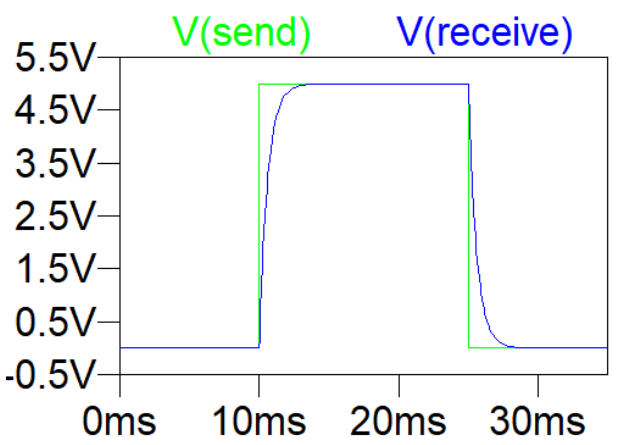

Fig. 8. Simulation Circuit (Touch Panel) 


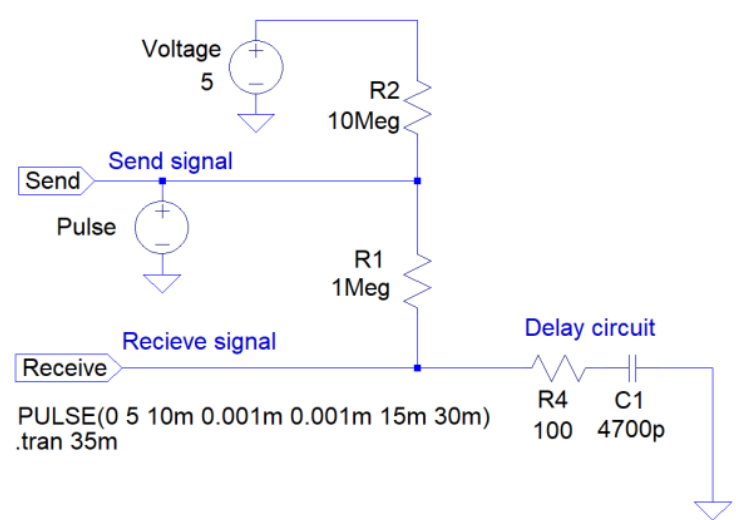

Fig. 9. Simulation Circuit (Push Button)

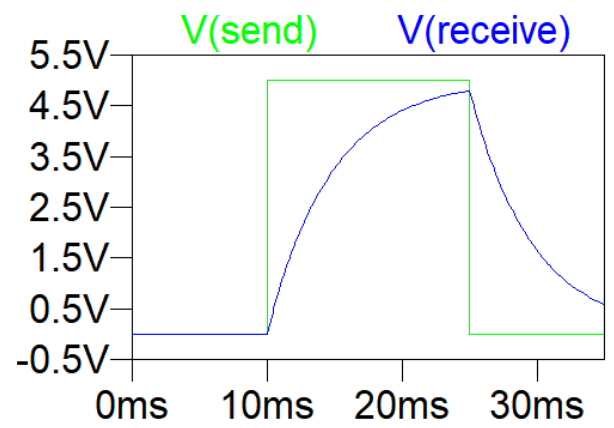

Fig. 10. Simulation Waveform (Push Button)

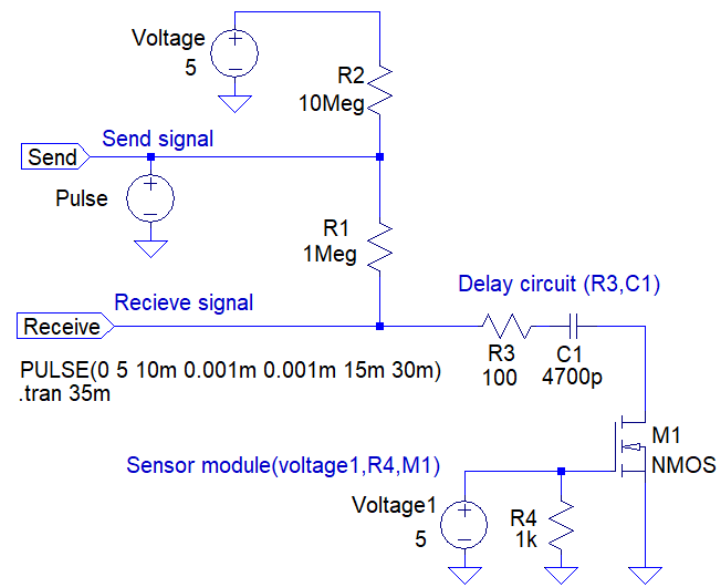

Fig. 11. Simulation Circuit (Sensor Module)

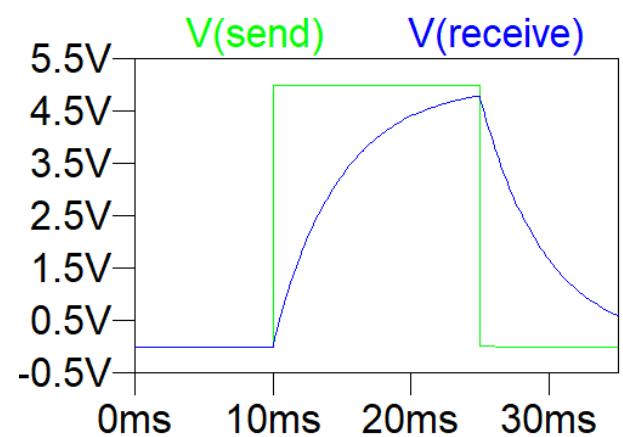

Fig. 12. Simulation Waveform (Sensor Module) determine whether the switch is on or off.

\subsection{Experiment}

Figure 13 shows the experimental circuit, and Figure 14 shows the circuit of the input part used in the experiment. In this experiment, a microcomputer (Arduino UNO Rev3) is used for the detection part, and I/O expanders (Microchip MCP23017) are used for the expanders. The I/O expander has the specification, in which a maximum number of connected I/O expanders is 8. Each $\mathrm{I} / \mathrm{O}$ expander has eight $\mathrm{I} / \mathrm{O}$ ports, so it can be connected to eight touch panels. The microcomputer has the role of master, and the I/O expanders have the role of slave. They communicate by $\mathrm{I} 2 \mathrm{C}$ communication each other. In this experiment, the voltage of the pulse signal sent by the output port is $5[\mathrm{~V}]$ and the human body is grounded. Figure 15 shows the waveform when there is no input. This means input is OFF. Figure 16 displays the waveform when touch panel is touched. Figure 17 is the waveform when a push button is pressed. Figure 18 shows the waveform when the sensor module outputs High. Figs. 16-18 correspond that input is ON. It can be seen that there is no delay in the received waveform compared to the send waveform when there is no input in Fig. 15, whereas there is a delay in the received waveform when there is an input in Figs. 16 - 18. This is also confirmed in the simulation in Chapter 4.2. Since the pulse width of the send pulse is 15 [ms], it takes about 15 [ms] to judge ON / OFF.

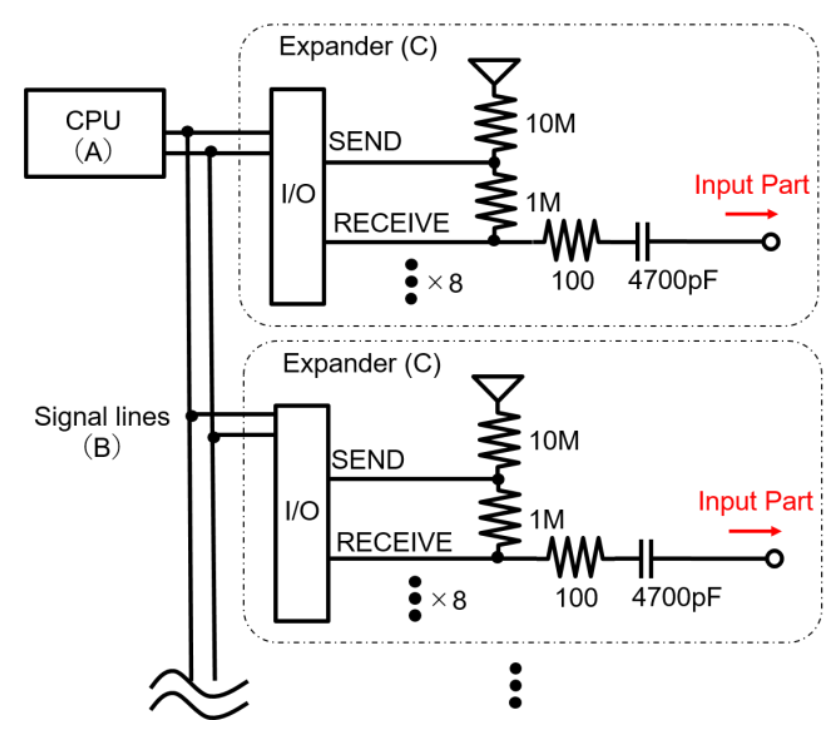

Fig. 13. Experimental Circuit (Detection Part) 

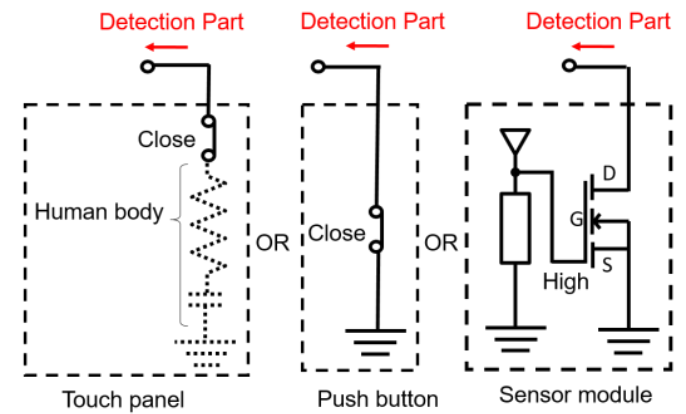

Fig. 14. Experimental Circuit (Input Part)

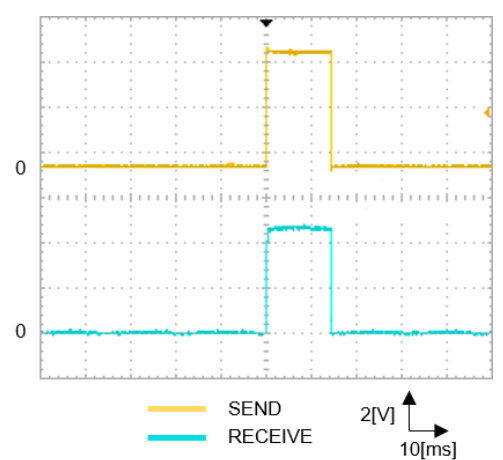

Fig. 15. Measurement Waveform (No Input:OFF)

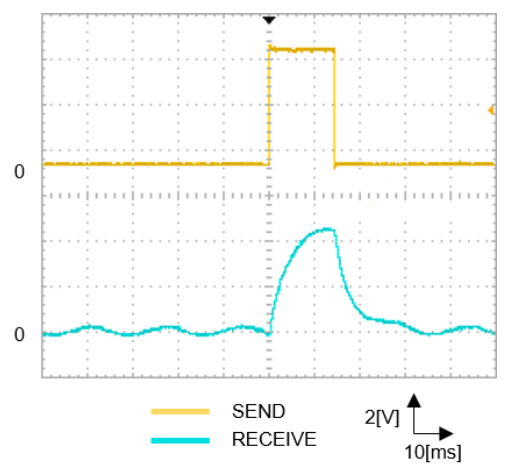

Fig. 16. Measurement Waveform (Touch Panel:ON)

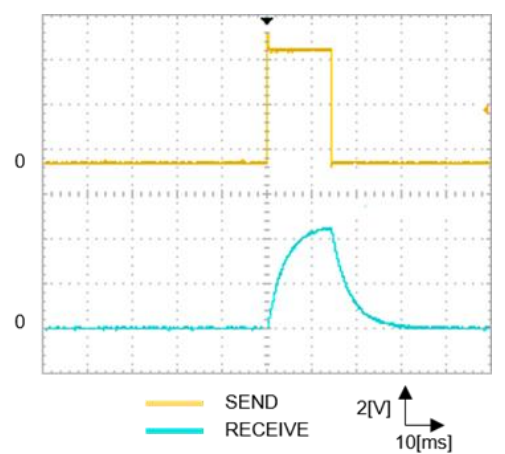

Fig. 17. Measurement Waveform (Push Button:ON)

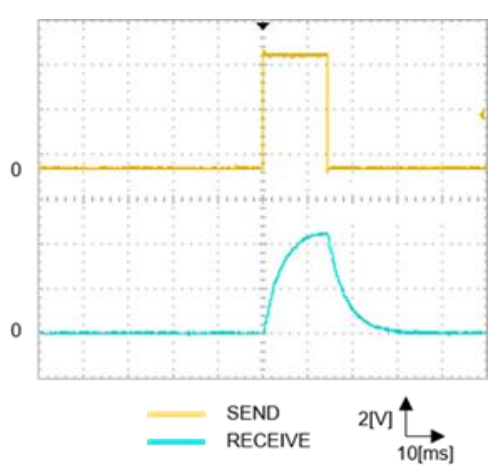

Fig. 18. Measurement Waveform (Sensor Module:ON)

influence of noise when the number of switches increases, and it is not possible to detect multiple touch panels at the same time. Furthermore, the type of is fixed as a touch type, and the input type can not be changed.

In this study, each switch is detected digitally and sequentially. As the result, the proposed method solves the number limitation of switches caused by the noise, and accurately detects the status of the multiple switches even if there are multiple inputs at the same time. In addition, the switch type can be changed from touch panel to pushbutton and sensor module. Regardless of the switch type, the same judgement algorithm and circuit is used. These have been confirmed by simulation and experiment. Even if the number of touch panels is increased, the proposed method can be detected with only two signal lines, and can easily change the design, shape, size, and surface condition. In addition, we can easily change the switch type by simply changing the terminals of the input device. Thus, the proposed method achieves a flexible input device with a higher degree of freedom.

\section{References}

(1) NXP Semiconductors(2014), "I2C-bus specification and user manual Rev.6"

(2) Seiichi Serikawa, Lifeng Zhang (2011), "Proposal of a touch panel switch with the function of bending and addition", 2nd International Conference on ICICI-BME, pp. 8-13

(3) Mingyu Fan, Daisuke Tanaka, Akira Yamawaki, Seiichi Serikawa (2015), "Simulation of touch sensor switch control circuit", The 3rd International Conference on ICIAE2015, pp. 223-226

\section{Conclusions}

The conventional method malfunctions due to the 